\title{
Uso de transplante de membrana amniótica no tratamento da ceratopatia bolhosa
}

\author{
Useofamniotic membrane transplantation to treat bullouskeratopathy
}

\author{
Daniel Keizo de Medeiros Haraguchi' \\ José Álvaro Pereira Gomes ${ }^{2}$ \\ Élcio Hideo Sato ${ }^{3}$ \\ Neil Ferreira Novo ${ }^{4}$ \\ Denise de Freitas ${ }^{5}$
}

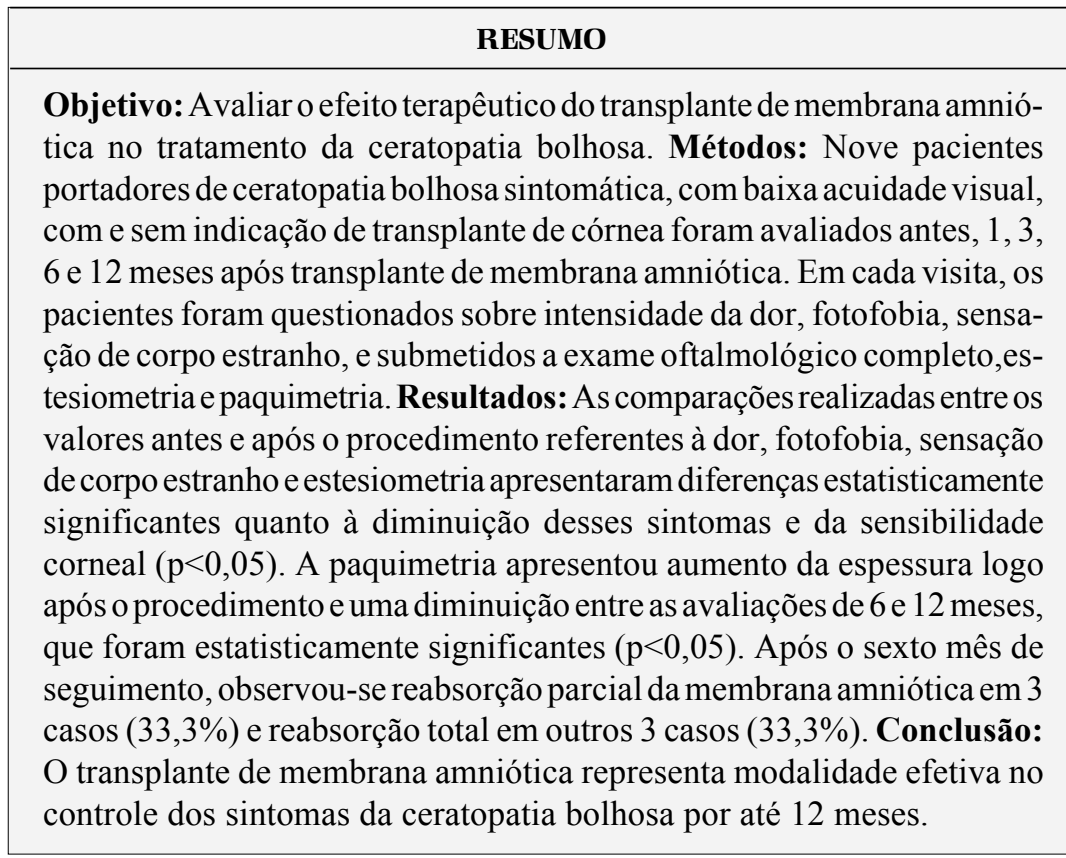

Descritores: Âmnio/transplante; Transplante heterotópico/utilização; Doenças da córnea/ cirurgia
Este trabalho foi realizado no Setor de Córnea e Doenças Externas do Departamento de Oftalmologia da Universidade Federal de São Paulo - UNIFESP.

Ex-residente do Departamento de Oftalmologia da Universidade Federal de São Paulo - UNIFESP;

2 Doutor em Oftalmologia e Colaborador do Setor de Córnea e Doenças Externas do Departamento de Oftalmologia da Universidade Federal de São Paulo - UNIFESP; 3 Doutor em Oftalmologia e Colaborador do Setor de Córnea e Doenças Externas do Departamento de Oftalmologia da Universidade Federal de São Paulo - UNIFESP; Diretor do Banco de Olhos do Hospital São Paulo;

${ }^{4}$ Professor Titular de Bioestatística e Medicina Preventiva da Faculdade de Medicina de Santo Amaro - UNISA; ${ }_{5}^{5}$ Professora Livre-Docente e Colaboradora do Setor de Córnea e Doenças Externas do Departamento de Oftalmologia da Universidade Federal de São Paulo UNIFESP.

Recebido para publicação em 23.10.2001 Aceito para publicação em 14.08.2002

Nota Editorial: Pela análise deste trabalho e por sua anuência na divulgação desta nota, agradecemos ao Dr. Sérgio Kwitko.

\section{INTRODUÇÃ̃O}

Ceratopatia bolhosa é uma doença caracterizada por edema do estroma corneano com formação de bolhas epiteliais e sub-epiteliais decorrente da descompensação do endotélio secundária a traumatismo cirúrgico, glaucoma não controlado, distrofias endoteliais como a distrofia de Fuchs e alterações congênitas ${ }^{(1)}$. Originam, freqüentemente, sintomas como diminuição da acuidade visual, dor, fotofobia e epífora ${ }^{(1)}$. Quando há bom potencial visual, o tratamento de escolha é o transplante penetrante de córnea que visa melhorar a visão e os sintomas, embora possa haver recorrência na córnea transplantada. Outras opções de tratamento incluem uso tópico de cloreto de sódio a 5\%, antiinflamatórios não hormonais, medicação antiglaucomatosa, lente de contato terapêutica, recobrimento conjuntival, cauterização e punções do estroma anterior ${ }^{(1-4)}$.

A membrana amniótica humana processada e preservada em banco de tecidos teve seu emprego em humanos aprovado pelo comitê de ética médica da Universidade Federal de São Paulo (UNIFESP) e tem sido utilizada para reconstrução de superfície ocular, mostrando resultados como facilidade de epitelização, redução da vascularização, cicatrização e inflama- 
ção $0^{(5-8)}$. O objetivo deste trabalho foi avaliar o efeito do transplante de membrana amniótica nos pacientes que apresentavam ceratopatia bolhosa sintomática.

\section{MÉTODOS}

Foram selecionados 9 pacientes seguidos no ambulatório de córnea e doenças externas do Departamento de Oftalmologia da UNIFESP, portadores de ceratopatia bolhosa, sintomáticos e que aguardavam transplante de córnea ou não tinham indicação cirúrgica, para estudo prospectivo.

Os pacientes foram avaliados previamente ao tratamento $\mathrm{e}$ $1,3,6$ e 12 meses após o tratamento. Os seguintes dados foram avaliados e graduados de acordo com a intensidade, de "0" a "3" cruzes: intensidade da dor; sensação de corpo estranho; fotofobia.

Além do questionário, foi realizada avaliação oftalmológica completa, que incluía: biomicroscopia detalhada, na qual foram observadas a superfície da córnea com e sem instilação tópica de fluoresceína, vascularização superficial e profunda, presença de bolhas epiteliais e sub-epiteliais; representação gráfica detalhada do quadro; fotografias à lâmpada de fenda (filme Kodak Professional E-200 Ektachrome Color Reversal Film, Lâmpada de fenda Hoya modelo HS); paquimetria ultrasônica (paquímetro Ultrasonic Pachometer Model 850, Humphrey Instruments, Inc); sensibilidade corneana medida pelo estesiômetro de (média de 3 medidas centrais conforme técnica preconizada). Todas as avaliações foram realizadas pelo mesmo examinador.

\section{Técnica cirúrgica}

A técnica de preservação da membrana já foi descrita anteriormente ${ }^{(5)}$. Uma carta de consentimento foi obtida de cada paciente. Todas as cirurgias foram realizadas pela mesma equipe. Para anestesia foi utilizada injeção peribulbar de cloridrato de bupivacaína a $0,5 \%$. Com auxílio de um trépano de 8 ou 9 $\mathrm{mm}$ de diâmetro (dependendo do tamanho da córnea), demarcava-se a área que era então submetida à remoção do epitélio, com lâmina de bisturi de número 15 . O mesmo trépano era utilizado para trepanar a membrana ainda sobre o papel de filtro. Separava-se a membrana do papel tomando o cuidado de manter a face estromal (mais irregular e menos brilhante) em contato com a córnea. Fixava-se a membrana na córnea com uma sutura contínua de 12 passagens eqüidistantes com fio nylon 10-0. Colocava-se uma lente de contato terapêutica que era mantida com uso de colírio de ofloxacina por 4 semanas, quando eram retirados os pontos.

\section{Análise estatística}

O teste para mudança de sinais de McNemar foi aplicado para determinarmos uma possível concordância entre a observação da variável na avaliação pré-operatória e na pós-operatória final (1 ano). Analisando as variáveis contínuas nos vários períodos de avaliação, utilizamos o teste não paramétri- co para "k" amostra não independente de Friedmann com o objetivo de evidenciar possíveis diferenças. O nível de rejeição estabelecido para a hipótese de nulidade foi sempre menor ou igual a $0,05(5 \%)$. Nas ocasiões em que a análise dos dados demonstrou-se estatisticamente significante, um asterisco $(*)$ foi colocado para caracterizá-la. Para os dados que não apresentaram significado estatístico utilizamos a sigla "ns" (não significante). As médias foram calculadas e apresentadas apenas a título de informação. Considerando-se que as variáveis não têm distribuição no padrão de curva de Gauss, não foi calculado o desvio padrão.

\section{RESULTADOS}

Foram analisados os resultados dos 9 pacientes que completaram o seguimento de 1 ano. Todas as cirurgias transcorreram sem intercorrências.

Do total, 6 pacientes eram do sexo feminino (67\%) e $3(33 \%)$ do sexo masculino. A idade variou de 24 a 84 anos (média de $57,9)$. As principais causas de ceratopatia bolhosa foram: pseudofacia (4 pacientes), afacia (1 paciente), falência secundária de transplante de córnea (1 paciente), trauma (1 paciente), falência secundária de transplante de córnea em olho pseudofácico (1 paciente), glaucoma em olho pseudofácico (1 paciente).

Todos os pacientes (100\%) apresentaram melhora da dor após 1 ano.

Em relação à fotofobia, 7 pacientes $(77,8 \%)$ relataram melhora e $2(22,2 \%)$ mantiveram-se inalterados após 1 ano, o que representa uma melhora estatisticamente significante $(p<0,05)$.

A sensação de corpo estranho também apresentou melhora significante $(p<0,05)$, com 7 pacientes referindo diminuição do sintoma, $1(11,1 \%)$ referindo piora e $1(11,1 \%)$ não indicando alteração.

Analisando a paquimetria, observamos nas comparações múltiplas um aumento da paquimetria média com diferença estatisticamente significante entre as avaliações inicial e de 1 mês, e inicial e de 3 meses $(p<0,05)$, e uma diminuição entre as avaliações de 1 mês e 6 meses, e de 1 mês e 1 ano $(p<0,05)$ (tabelas $1 \mathrm{e}$ 2).

\begin{tabular}{|c|c|c|c|c|c|}
\hline \multirow[b]{2}{*}{ Pacientes } & \multirow[b]{2}{*}{ Pré } & \multicolumn{4}{|c|}{ Paquimetria média $(\mathrm{mm})$} \\
\hline & & 1 mês & 3 meses & 6 meses & 12 meses \\
\hline 1 & 873 & 956 & 947 & 900 & 856 \\
\hline 2 & 852 & 1015 & 1001 & 926 & 954 \\
\hline 3 & 976 & 1068 & 1056 & 1031 & 994 \\
\hline 4 & 915 & 1038 & 998 & 968 & 942 \\
\hline 5 & 782 & 878 & 864 & 844 & 840 \\
\hline 6 & 859 & 972 & 928 & 939 & 926 \\
\hline 7 & 840 & 994 & 974 & 677 & 686 \\
\hline 8 & 815 & 954 & 916 & 840 & 837 \\
\hline 9 & 982 & 1044 & 985 & 954 & 968 \\
\hline médias & 877 & 991 & 963 & 998 & 889 \\
\hline
\end{tabular}




\begin{tabular}{|c|c|c|}
\hline $\begin{array}{c}\text { Comparações } \\
\text { múltiplas }\end{array}$ & Estesiometria & Paquimetria média \\
\hline 0 vs 1 & * & * \\
\hline 0 vs 3 & ns & * \\
\hline 0 vs 6 & ns & ns \\
\hline 0 vs 12 & ns & ns \\
\hline 1 vs 3 & ns & ns \\
\hline 1 vs 6 & ns & * \\
\hline 1 vs 12 & ns & * \\
\hline 3 vs 6 & ns & ns \\
\hline 3 vs 12 & ns & ns \\
\hline 6 vs 12 & ns & ns \\
\hline \multicolumn{3}{|c|}{ "“”indica $p<0,05$; "ns"= não significante } \\
\hline
\end{tabular}

A estesiometria mostrou uma diferença estatisticamente significante apenas entre as avaliações inicial e de 1 mês.

À biomicroscopia, foi observado que a membrana adere-se bem à superfície da córnea, com transparência maior no centro do que na periferia e apresentando aspecto estético bastante satisfatório (Figuras 1a e 1b). Após 1 ano de seguimento, observou-se que dos 9 casos, $3(33,3 \%)$ apresentaram manutenção, $3(33,33 \%)$ apresentaram reabsorção parcial e 3 $(33,33 \%)$ apresentaram reabsorção total da membrana amniótica transplantada. (Figuras $2 \mathrm{a}$ e $2 \mathrm{~b}$ ). Nenhum paciente apresentou alteração da vascularização corneana.

A acuidade visual manteve-se inalterada em 8 pacientes $(88,9 \%)$ e melhorou em 1 paciente, o que não representou alteração significante.

\section{DISCUSSÃO}

Os portadores de ceratopatia bolhosa têm, freqüentemen-

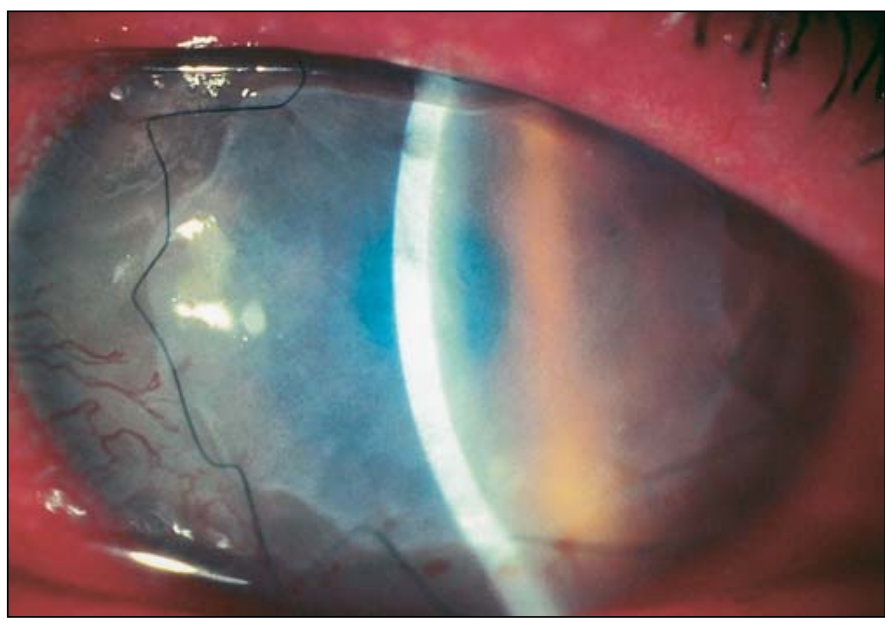

Figura 1a - Aspecto biomicroscópico da membrana amniótica transplantada em um caso de ceratopatia bolhosa após 1 mês da cirurgia. Nota-se bom aspecto estético te, sua qualidade de vida prejudicada pelo desconforto causado pelos sintomas da doença. Há, na literatura, diversos tratamentos descritos como alternativas para estes casos. O uso de esteróides e agentes hiperosmóticos é preconizado por alguns autores, mas apresenta resultados limitados nos casos avançados $^{(1)}$. A lente de contato terapêutica mostra-se eficaz, porém está associada ao aumento da vascularização e do risco de infecção quando utilizada por tempo prolongado ${ }^{(1-2)}$. Punções do estroma anterior da córnea representam um prático e eficiente procedimento para o tratamento dos sintomas da ceratopatia bolhosa ${ }^{(3)}$. Porém, necessita ser repetido em 1/3 dos $\operatorname{casos}^{(3)}$. Outros procedimentos mais invasivos incluem a cauterização da membrana de Bowman, que pode induzir modificações acentuadas na curvatura da córnea, vascularização ou necrose estromal, prejudicando um possível transplante de córnea no futuro $^{(1)}$. E o recobrimento conjuntival, que resulta em um aspecto estético muitas vezes insatisfatório, além de causar deficiência de células limbares, o que também dificulta um transplante de córnea ${ }^{(9-10)}$.

Nesse estudo, pudemos comprovar a eficiência do transplante de membrana amniótica como alternativa no tratamento da ceratopatia bolhosa. Nossos resultados assemelham-se aos descritos por Pires et al., que observou ausência de dor em 90\% de pacientes com ceratopatia bolhosa submetidos a esse procedimento $^{(11)}$. Além do excelente resultado estético, a transparência que a membrana apresenta quando aderida à córnea permitiu a manutenção da acuidade visual em 8 pacientes e melhora em 1.

O aumento da espessura média na avaliação de 1 mês deve-se, presumivelmente à adição de tecido própria da técnica, enquanto que a diminuição posterior nas avaliações de 6 meses e 1 ano, coincidindo com o aparente desaparecimento da membrana de algumas áreas da córnea em alguns casos, pode ser explicada por uma dissolução ou talvez integração da mesma na córnea. Possivelmente, a análise histopatológica dessas córneas possa ser útil para analisar o fato.

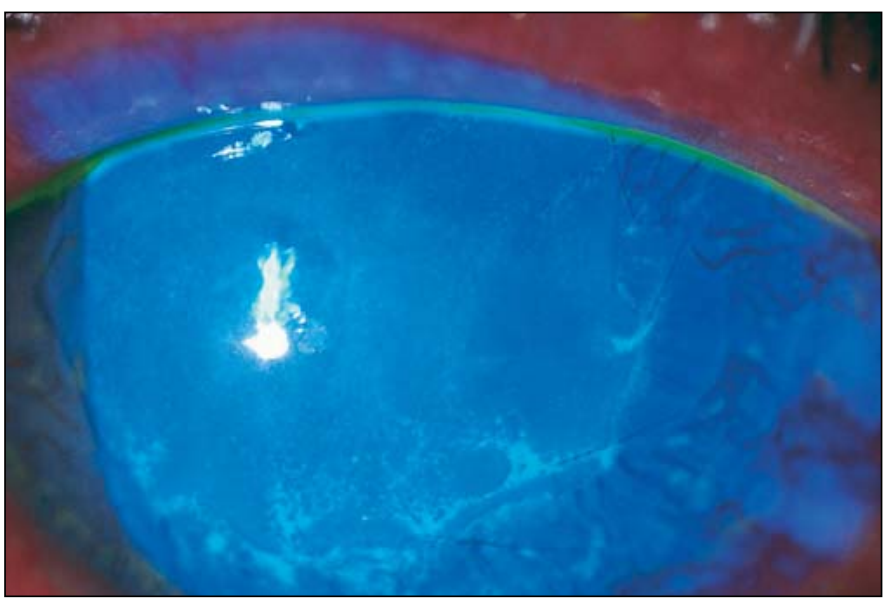

Figura 1b - Aspecto biomicroscópico com utilização de filtro azul de cobalto do mesmo caso da figura 1a após instilação de fluoresceína sódica $1 \%$. Note a regularidade da superfície da córnea e ausência de defeito epitelial 


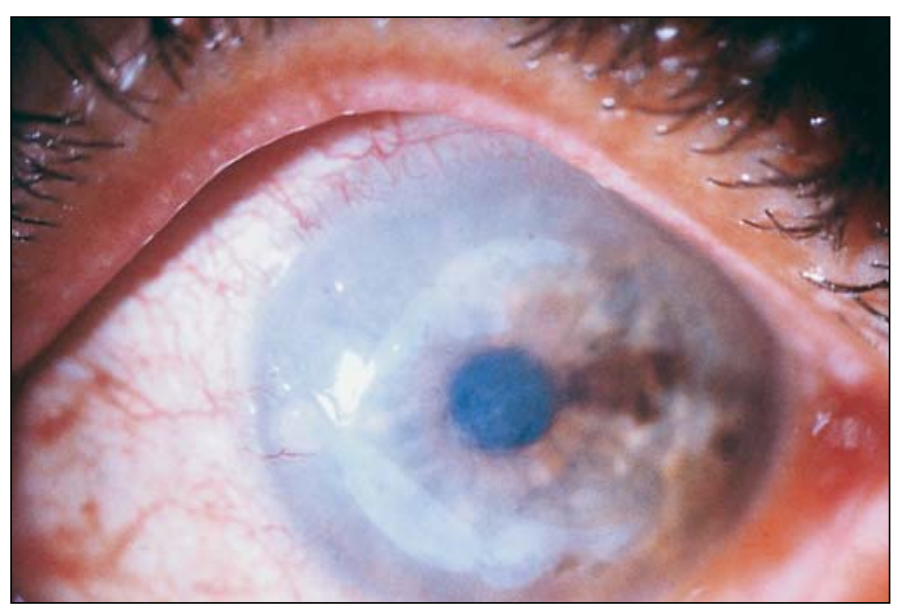

Figura 2a - Aspecto biomicroscópico da membrana amniótica parcialmente reabsorvida 6 meses após o transplante

O efeito benéfico da membrana amniótica na ceratopatia bolhosa pode ser atribuído a adição de novo substrato, composto pela membrana basal e matriz estromal avascular da membrana amniótica ${ }^{(11)}$. Além disso, apresenta propriedades estimuladoras da epitelização e moduladoras dos processos cicatricial e inflamatório. Estudos prospectivos e comparativos são necessários para melhor definirmos sua utilização na ceratopatia bolhosa.

\section{CONCLUSÕES}

A técnica do transplante de membrana amniótica mostrouse eficaz no controle dos sintomas da ceratopatia bolhosa, particularmente da dor pelo menos por 1 ano pós-operatório.

Apresenta ainda, aspecto estético satisfatório e a manutenção da acuidade visual.

Deve ser considerada uma alternativa para o tratamento desses pacientes.

\section{ABSTRACT}

Purpose: To evaluate the therapeutic effect on amniotic membrane transplantation in symptomatic bullous keratopathy. Methods: Nine patients with symptomatic bullous keratopathy, candidates or not for penetrating keratoplasty were evaluated before, 1, 3, 6 and 12 months after amniotic membrane transplantation. During each visit, patients have been asked about pain, photophobia, and foreign body sensation and were submitted to complete ophthalmological evaluation, esthesiometry and ultrasound pachymetry. Results: Comparing evaluations performed previously and after the procedure regarding pain, photophobia, foreign body sensation and esthesiometry, there was a statistical significant reduction in symptoms and corneal sensitivity. Medium pachymetry showed an increased thickness soon after the procedure and

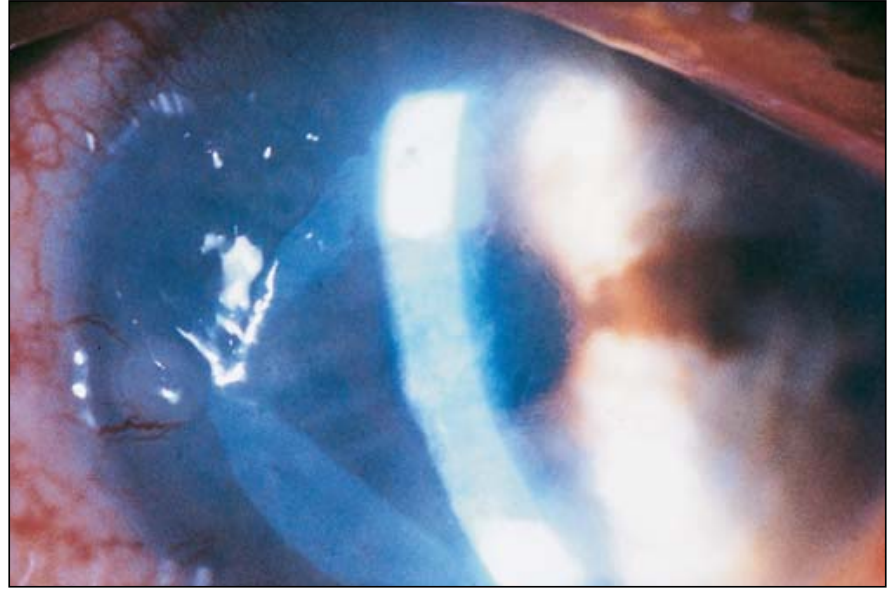

Figura 2b - Corte biomicroscópico do mesmo caso da figura 2a. Note que a reabsorção parcial da membrana amniótica ocorreu principal mente na área central

decreasing between 6 and 12 months. It was observed that after 6 months of follow-up, the transplanted amniotic membrane became partially dissolved in 3 cases $(33.3 \%)$ and totally dissolved in other 3 cases $(33.3 \%)$. Conclusion: Amniotic membrane transplantation represents an effective alternative for the treatment of symptomatic bullous keratopathy up to 12 months of follow-up.

Keywords: Amnion/transplantation; Transplantation, heterotopic/utilization; Corneal diseases/surgery

\section{REFERÊNCIAS}

1. Aquavella JV. Corneal edema. In: Leibowitz HM, editor. Corneal disorders: Clinical diagnosis and management. Philadelphia: WB Saunders; 1984.p.164-82.

2. Plotnik RD, Mannis MJ, Schwab IR. Therapeutic contact lenses. Int Ophthalmol Clin 1991;31:35-52.

3. Gomes JAP, Haraguchi DKM, Zambrano DU, Villavicencio LI, Cunha MC, Freitas D. Punções do estroma anterior no tratamento da ceratopatia bolhosa. Arq Bras Oftalmol 2000;63:133-7.

4. Cormier G, Brunette I, Boisjoly HM, LeFrançois M, Shi ZH, Guertin MC. Anterior stromal punctures for bullous keratopathy. Arch Ophthalmol 1996;114:654-8.

5. Gomes JAP, Komagome CM, Santos N, Cunha MC, Freitas D. Membrana amniótica nas cirurgias reconstrutivas da superfície ocular nas ceratoconjuntivites cicatriciais. Arq Bras Oftalmol 1999;62:562-76.

6. Tsubota K, Satake Y, Ohyama M, Toda I, Takano Y, Ono M et al. Surgical reconstruction of the ocular surface in advanced ocular cicatricial pemphigoid and Stevens-Johnson syndrome. Am J Ophthalmol 1996;122:38-52.

7. Azuara-Blanco A, Pillai CT, Sarhan A, Dua HS. Amniotic membrane transplantation for ocular surface reconstruction. Invest Ophthalmol Vis Sci 1998;39(4 Suppl):S428.

8. Tseng SC, Prabhasawat P, Barton K, Gray T, Meller D. Amniotic membrane transplantation with or without limbal allografts for corneal surface reconstruction in patients with limbal stem cell deficiency. Arch Ophthalmol 1998;116:431-41.

9. Smith R. Surgical relief of bullous keratopathy (Gunderson's operation). Trans Ophthalmol Soc U K 1965;85:307-16.

10. Alino AM, Perry HD, Kanellopoulos AJ, Donnenfeld ED, Rahn EK. Conjunctival flaps. Ophthalmology 1998;105:1120-3.

11. Pires RT, Tseng SC, Prabhasawat P, Puangsricharern V, Maskin SL, Kim JC, et al. Amniotic membrane transplantation for symptomatic bullous keratopathy. Arch Ophthalmol 1999;117:1291-7. 